\title{
Significance of lymphocytosis in bronchoalveolar lavage in suspected ocular sarcoidosis
}

\author{
T. Takahashi*, A. Azuma*, S. Abe*, O. Kawanami”, K. Ohara", S. Kudoh*
}

Significance of lymphocytosis in bronchoalveolar lavage in suspected ocular sarcoidosis. T. Takahashi, A. Azuma, S. Abe, O. Kawanami, K. Ohara, S. Kudoh. (C) ERS Journals Ltd 2001

ABSTRACT: Ocular sarcoidosis is frequent in Japan, but in many cases the condition remains undiagnosed in patients with suspected ocular sarcoidosis. Bronchoalveolar lavage (BAL) was performed in order to study the clinical implications of lymphocytosis of BAL fluid in such patients with characteristic ocular manifestations.

The subjects included in this study were 39 patients with suspected ocular sarcoidosis. The patients were divided into four types based on high-resolution computed tomography (HRCT) findings; no lung involvement (HRCT-0), bilateral hilar lymphadenopathy (BHL) without lung involvement (HRCT-I), lung involvement and BHL (HRCT-II), and lung involvement and no BHL (HRCT-III). Transbronchial lung biopsy (TBLB) and BAL were conducted after examining serum angiotensin-converting enzyme and serum lysozyme values, skin test for purified protein derivative chest radiograph, HRCT, and gallium scintigram.

Twenty patients were histologically diagnosed as having sarcoidosis, and 19 patients remained undiagnosed. Granuloma was identified by TBLB in 19 of 20 patients in type HRCT-II but in only one of 19 patients in types HRCT-0 and HRCT-I $(p<0.0001)$. Lymphocytosis in BAL $(>15 \%)$ was identified in all patients who showed lung field involvement (type HRCT-II) and in 16 of 19 patients without lung field involvement (types HRCT-0 and HRCT-I). There were 10 patients whose only relevant findings were lymphocytosis in BAL. Among these 10 patients, an increased CD4+/CD8+ ratio $(>3.5)$ in BAL was seen in $60 \%$.

The authors conclude that high-resolution computed tomography results yield the same degree of diagnostic accuracy as transbronchial lung biopsy in ocular sarcoidosis suspects. However, bronchoalveolar lavage revealed significant lymphocytosis in patients with negative high-resolution computed tomography results. It should be kept in mind that a diagnostic group of patients with sarcoidosis who manifest ocular involvement and lymphocytosis in bronchoalveolar lavage exists.

Eur Respir J 2001; 18: 515-521.
*Fourth Dept of Internal Medicine Nippon Medical School, Tokyo, ${ }^{\#}$ Dept of Molecular Pathology Institute of Gerontology Nippon Medical School, Kawasaki, and "Dept of Ophthalmology, Nippon Medical School, Tokyo, Japan.

Correspondence: A. Azuma

1-1-5 Sendagi

Bunkyo-ku

Tokyo 113-8603

Japan

Fax: 81356853075

Keywords: Bronchoalveolar lavage high-resolution computed tomography lymphocytosis ocular sarcoidosis sarcoidosis

Received: December 91999 Accepted after revision May 122001
Sarcoidosis is a systemic granulomatous disorder of unknown aetiology involving various organs. Intraocular tissues are frequently involved in patients with sarcoidosis in the Japanese population, and over half of the patients developed ocular inflammation as an initial clinical manifestation of the disease [1]. The intraocular lesions are highly characteristic, suggesting the diagnosis of sarcoidosis, and ophthalmologists send the patients to Depts of Respiratory Diseases for systemic survey. It is, however, often difficult to establish the diagnosis and to confirm the lesions as sarcoidosis. This is probably due to the limited volume of intraocular granulation tissue, which is not well detected by results of systemic examinations, such as an increased serum angiotensin-converting enzyme (SACE) level. Ophthalmology detects cellular abnormalities and minimal granuloma as aqueous cells, iris nodules, trabecular nodules and retinal perivasculitis using in vivo biomicroscopy including slit-lamp and ophthalmoscopy. Ocular lesions may precede macroscopic granuloma formation in extraocular organs.

Transbronchial lung biopsy (TBLB) is a useful procedure for a definitive diagnosis, but its diagnostic yield in sarcoidosis ranges 60-97\% [2-4]. Such differences in diagnostic yield are thought to be due to the number of samples, sites of biopsy, and differences in the stage of the disease [3, 4]. Roentgenographic detection of lung field involvement is important for the pathological diagnosis of granuloma, and high-resolution computed tomography (HRCT) has been shown to be useful for deciding biopsy sites [5].

In the present study, bronchoalveolar lavage (BAL) and TBLB were performed in ocular sarcoidosis suspects to study the clinical relevance of lymphocytosis in BAL fluid for the diagnosis of ocular sarcoidosis. HRCT of the chest was also performed to assess the lung involvement, and to compare the validity of lymphocytosis in BAL and HRCT. 


\section{Material and methods}

\section{Subjects}

Subjects in the present study were 39 patients with suspected ocular sarcoidosis. All but one patient had normal pulmonary function test results (table 1) and all patients underwent blood tests, chest radiography, HRCT, TBLB and BAL. Ocular sarcoidosis was suspected based on one or more of the following typical findings: granulomatous iritis with mutton-fat keratic precipitates or iris nodules, trabecular nodules, tent-like peripheral anterior synechiae, snowball or string-of-pearls vitreous opacities, retinal perivasculitis, or spotty retinochoroidal exudates. Most of patients had at least two such lesions. However, the number of these findings was not correlated with the detection rate of granuloma by TBLB [6]. Based on chest radiographs, the patients were divided into four stages in accordance with American Thoracic Society chest radiographic staging (0, I, II, and III) [7]. All subjects gave informed consent to participate in the study.

\section{High-resolution computed tomography}

HRCT examination was performed with an XVigor scanner (Toshiba Co., Tokyo, Japan) at $150 \mathrm{mAs}$ and $120 \mathrm{kV}$. During scanning, patients were instructed to hold their breath at the maximal inspiration level.

Thin section radiographs ( $2 \mathrm{~mm}$ section thickness) were taken from the apex to the base of the lung in increments of $10 \mathrm{~mm}$ with the patient in a dorsal position. Scans were reconstructed via a high spatial frequency reconstruction algorithm and viewed at

Table 1. - Characteristics of ocular sarcoidosis suspects

\begin{tabular}{lc}
\hline Patients n & 39 \\
Age yrs & \\
$<40$ & $18(46)$ \\
$\geqslant 40$ & $21(54)$ \\
Sex & $12(31)$ \\
M & $27(69)$ \\
F & $14(36)$ \\
Smoking & $25(64)$ \\
Current & \\
Negative & $2(5)$ \\
Other involved organ & $4(10)$ \\
Heart & $1(3)$ \\
Skin & \\
Liver & $5(13)$ \\
Systemic therapy & $2(5)$ \\
Prednisolone & $38(97)$ \\
ACE antagonist & $1(3)$ \\
Pulmonary function test results* & \\
Normal & \\
Obstructive &
\end{tabular}

Data are presented as $\mathrm{n}(\%)$. M: male; F: female; ACE: angiotensin-converting enzyme. *: forced expiratory volume in one second $\left(\mathrm{FEV}_{1}\right) \%$ pred $>70 \%$, forced vital capacity (FVC) $\%$ pred $>70 \%$, transfer factor of the lung for carbon monoxide $(T \mathrm{~L}, \mathrm{CO})<70 \%$ pred; ${ }^{\#}$ : ratio $<0.7$. windows appropriate for pulmonary parenchyma and for mediastinum (level and width differed for each patient).

Pulmonary involvement was determined by more than three respiratory physicians. Typical findings of lung involvement were: 1) thickened bronchovascular bundles; 2) spherical, mass-like opacities; 3) multiple, discrete, small nodules; 4) thickening of the pleural surface; and 5) ground glass opacities [8-10]. If the largest diameter of a lymph node was $>10 \mathrm{~mm}$, lymphadenopathy was diagnosed. Subjects were divided into four types according to the HRCT findings as follows: HRCT-0, no abnormal findings; HRCT-I, hilar and/or mediastinal lymphadenopathy; HRCT-II lymphadenopathy with lung field involvement; and HRCT-III, lung field involvement without lymphadenopathy.

Bronchoscopy, bronchoalveolar lavage, transbronchial lung biopsy

After premedication with atropine and hydroxyzine, a bronchoscope (BF10, Olympus, Tokyo, Japan) was inserted under local anaesthesia with lidocaine and wedged in the right middle lobe. BAL was performed three times in a single lung segment (right S4 or S5) with $30 \mathrm{~mL} \times 1$ and $50 \mathrm{~mL} \times 2$ of sterile $0.9 \%$ saline. Cells centrifuged with a Cytocentrifuge (Shandon Products, Cheshire, UK) were stained on slides by May-Grunwald Giemsa and cell populations were assessed in units of 500 cells. BAL fluid was analysed for the ratio of CD4+/CD8+ T-cells (using fluorescence labelled monoclonal antibodies and flow cytometrical analysis) by S.R.L. Corp. (Tokyo, Japan). The authors also attempted to determine the presence of tuberculosis and other infectious diseases by culture methods.

Alligator forceps were used via the bronchoscope to obtain five biopsies from the bronchial branches B2b, $\mathrm{B} 3 \mathrm{a}$ and $\mathrm{B} 8 \mathrm{a}$. If infiltrations and/or fine granular shadows seen on HRCT were found in another part of the lung, those areas were focused upon for biopsy specimens.

\section{Other examinations}

Serum examinations, including those for serum angiotensin converting enzyme (SACE) and serum lysozyme (LZM), skin test for purified protein derivative (PPD), and gallium (Ga) scintigram were conducted to assist in the diagnosis of sarcoidosis.

\section{Statistical analysis}

Statistical assessments were made using Fisher's exact probability test; a p-value $<0.01$ was considered statistically significant. 


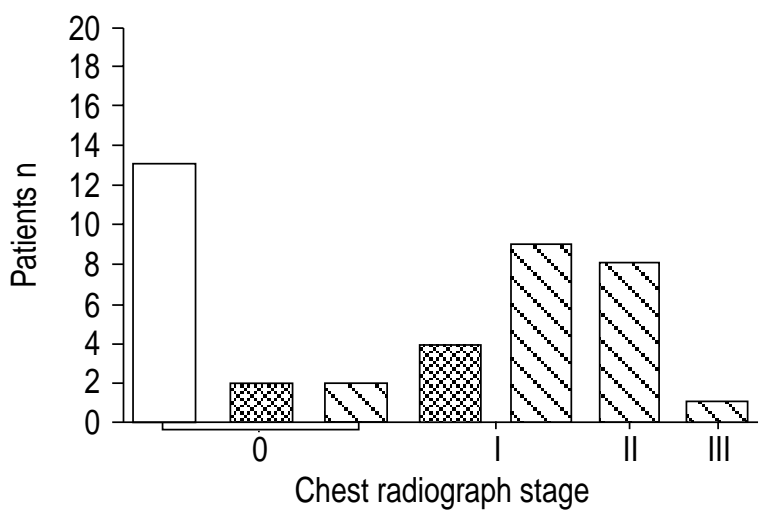

Fig. 1.-Comparison of chest radiograph stages and high-resolution computed tomography (HRCT) types. $\square$ : no abnormal findings on HRCT indicating type-0; : hilar and/or mediastinal lymphadenopathy on HRCT, type-I; $\nabla$ : lymphadenopathy with lung field involvement, type HRCT-II. Two of 17 patients with stage 0 , nine of 13 with stage I and one with stage III were categorized as type HRCT-II. Two of 17 patients with stage 0 were classified as type HRCT-I. Thirteen patients were typed as HRCT- 0 , nine were typed as HRCT-I, and 20 were typed as HRCT-II. No patients were typed as HRCT-III (lung field involvement without lymphadenopathy).

\section{Results}

Twenty patients were histologically diagnosed as having sarcoidosis, and 19 patients remained undiagnosed.

The pulmonary involvement of ocular sarcoidosis defined by HRCT was compared with that defined by conventional chest radiograph (fig. 1). Two of 17 patients with stage 0 and 9 of 13 patients with stage I were categorized as type HRCT-II, showing subtle abnormal shadows in the lung field. One patient with type HRCT-III was found to have lymphadenopathy by HRCT (type HRCT-II). Two of 17 patients with stage 0 were classified as type HRCT-I with mediastinal lymphadenopathy detected by HRCT but not by radiograph. Those patients were then categorized according to HRCT findings, as shown in figure 1 . According to HRCT typing, the diagnostic yield of TBLB (fig. 2) was compared between type HRCT-II and types HRCT-0 and -I. Granuloma was identified by TBLB in 19 of 20 patients with type HRCT-II as opposed to one of 19 patients with types HRCT-0 and -I; this difference was statistically significant $(p<0.0001)$. It could not be said, however, that it was a typical sarcoid granuloma in the patient with type HRCT-I. Lung field involvement and mediastinal lymphadenopathy was found on HRCT in all patients in whom sarcoid granuloma was detected by TBLB, but the findings by TBLB were inconsistent with those by conventional chest radiograph.

Lymphocytosis $>15 \%$ in BAL was identified in all patients with type HRCT-II and in 16 of 19 patients with types HRCT-0 and -I (fig. 2). Morphological characteristics of lymphocytes were very similar to those of patients who were histologically diagnosed as having sarcoidosis, because all the lymphocytes were small and well matured (data not shown). None of the
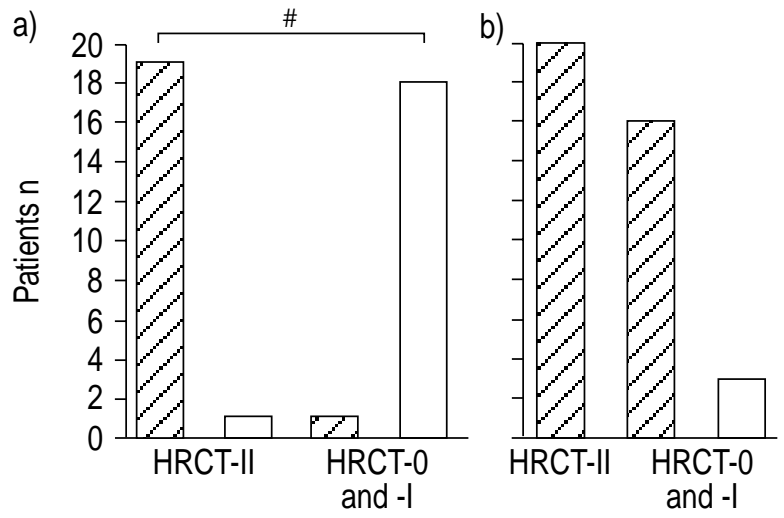

Fig. 2.-Comparison of positive ratio in a) transbronchial lung biopsy (TBLB) and b) bronchoalveolar lavage (BAL) between high-resolution computed tomography (HRCT) types -0 and -I and type -II. $\nabla$ : patients identified with granuloma in a) and those with lymphocytosis $(>15 \%)$ in BAL in b). $\square$ : patients not identified with granuloma in a) and with lymphocytosis $>15 \%$ in b). In BAL, $>15 \%$ was seen not only in type HRCT-II (all patients), but also in types HRCT-0 and -I (16 of 19 patients). This indicates that BAL cell lymphocytosis is an early manifestation of ocular sarcoidosis in the radiographically intact lung field. ${ }^{\#}$ : $\mathrm{p}<$ 0.0001 , type HRCT-II as compared to types HRCT-0 and -I.

BAL fluid showed any infectious diseases by culture methods.

These types were then compared with regard to the CD4+/CD8+ ratio in BAL (fig. 3). An elevated CD4+/ $\mathrm{CD} 8+$ ratio $(>3.5)$ in $\mathrm{BAL}$ was seen in $\geqslant 60 \%$ of the cases examined, with no significant difference among the types. Three patients did not have lymphocytosis or any other findings suggesting sarcoidosis, except the typical picture of ocular sarcoidosis (table 2). However, one patient (patient number 39) exhibited an elevated $\mathrm{CD} 4+/ \mathrm{CD} 8+$ ratio of 6.4 . In some cases, minimal changes in computed tomography findings in a limited subpleural area (fig. 4a) were identified among the pathological features of TBLB specimens (fig. 4b).

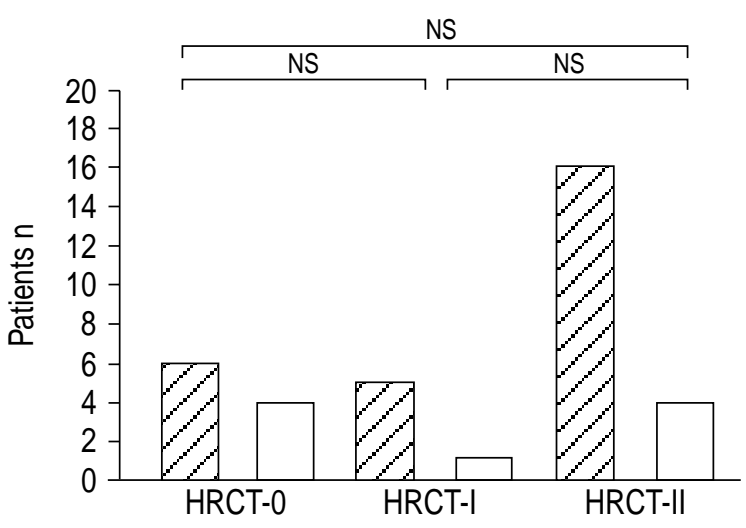

Fig. 3. - Comparison of CD4+/CD8+ ratio of $>3.5 \nabla$ : in bronchoalveolar lavage cell lymphocytosis between types oriented by high-resolution computed tomography (HRCT). $\square$ : patients with a CD4+/CD8+ ratio of $<3.5$. HRCT-0: no abnormal findings $(\mathrm{n}=10)$; HRCT-I: hilar and/or mediastinal lymphadenopathy $(\mathrm{n}=6)$; HRCT-II: lymphadenopathy with lung field involvement $(n=20)$. NS: nonsignificant. Positive ratio in each stage was not significantly different. 
Table 2. - Characteristics of 39 ocular sarcoidosis suspects

\begin{tabular}{|c|c|c|c|c|c|c|c|c|c|c|c|}
\hline \multirow{2}{*}{$\begin{array}{l}\text { Patient } \\
\text { no. }\end{array}$} & \multirow[t]{2}{*}{ Sex } & \multirow{2}{*}{$\begin{array}{l}\text { Age } \\
\text { yrs }\end{array}$} & \multirow{2}{*}{$\begin{array}{l}\text { Radiograph } \\
\text { stage }\end{array}$} & \multirow{2}{*}{$\begin{array}{l}\text { HRCT } \\
\text { type }\end{array}$} & \multirow{2}{*}{$\begin{array}{c}\text { Serum LZM } \\
\mu \mathrm{g} \cdot \mathrm{mL}^{-1}\end{array}$} & \multirow{2}{*}{ 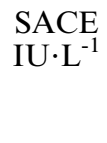 } & \multirow{2}{*}{$\begin{array}{c}\text { PPD } \\
\text { skin test }\end{array}$} & \multirow{2}{*}{$\begin{array}{l}\text { Ga scinti- } \\
\text { gram }\end{array}$} & \multirow{2}{*}{$\begin{array}{l}\text { TBLB } \\
\text { granuloma }\end{array}$} & \multicolumn{2}{|l|}{ BAL } \\
\hline & & & & & & & & & & Lymphocyte & $\begin{array}{l}\mathrm{CD} 4+/ \\
\mathrm{CD} 8+\end{array}$ \\
\hline 1 & M & 35 & III & II & $20.2^{*}$ & $25.2^{\#}$ & - & + & + & $62^{\circ}$ & $3.7^{+}$ \\
\hline 2 & $\mathrm{~F}$ & 40 & II & II & $24^{*}$ & $32^{\#}$ & - & + & + & $88^{\circ}$ & $13.6^{+}$ \\
\hline 3 & M & 53 & II & II & $18.5^{*}$ & $24.9^{\#}$ & - & + & + & $67^{\circ}$ & $12.3^{+}$ \\
\hline 4 & M & 51 & II & II & $15.9^{*}$ & $23.5^{\#}$ & - & + & + & $63^{\circ}$ & $7.4^{+}$ \\
\hline 5 & $\mathrm{~F}$ & 67 & II & II & $11.6^{*}$ & 19 & - & + & + & 52 & $6.66^{+}$ \\
\hline 6 & $\mathrm{M}$ & 28 & II & II & $37.9 *$ & 41 & ND & + & + & $48^{\circ}$ & 2.68 \\
\hline 7 & $\mathrm{M}$ & 41 & II & II & $14.5^{*}$ & $31.7^{\#}$ & + & + & + & 38 & 2.49 \\
\hline 8 & $\mathrm{~F}$ & 57 & II & II & $17.2^{*}$ & 9.6 & - & + & + & $31^{\circ}$ & $6.52^{+}$ \\
\hline 9 & M & 26 & II & II & $13.3^{*}$ & 8 & - & ND & + & $27^{\bullet}$ & $4.7^{+}$ \\
\hline 10 & $\mathrm{~F}$ & 60 & I & II & 1.9 & 14.9 & - & + & + & $63^{\circ}$ & $6.89^{+}$ \\
\hline 11 & $\mathrm{~F}$ & 68 & I & II & ND & $25.8^{\#}$ & - & + & + & $61^{\bullet}$ & $33^{+}$ \\
\hline 12 & M & 26 & I & II & 9.3 & 13.9 & + & + & + & $53^{\circ}$ & $6.83^{+}$ \\
\hline 13 & $\mathrm{~F}$ & 72 & I & II & $12.8^{*}$ & $23.4^{\#}$ & + & ND & + & $49^{\bullet}$ & 1.93 \\
\hline 14 & $\mathrm{~F}$ & 60 & I & II & 8.7 & $24.9^{\#}$ & - & + & + & $45^{\star}$ & $8.34^{+}$ \\
\hline 15 & $\mathrm{M}$ & 24 & I & II & $11.1^{*}$ & 16.2 & + & - & + & $27^{\bullet}$ & $4.89^{+}$ \\
\hline 16 & M & 36 & I & II & 6.7 & 10.7 & - & - & - & $19^{\circ}$ & $4.77^{+}$ \\
\hline 17 & $\mathrm{~F}$ & 40 & I & II & 7 & 17 & + & + & + & $15^{\circ}$ & $3.54^{+}$ \\
\hline 18 & $\mathrm{~F}$ & 34 & I & II & ND & 17.7 & + & + & + & $15^{\circ}$ & 2.83 \\
\hline 19 & $\mathrm{~F}$ & 64 & I & I & $12.1^{*}$ & $27^{\#}$ & - & + & - & $49^{\circ}$ & $12.6^{+}$ \\
\hline 20 & $\mathrm{~F}$ & 26 & I & I & $13.3^{*}$ & 16.1 & - & ND & - & $43^{\bullet}$ & $4.89^{+}$ \\
\hline 21 & $\mathrm{~F}$ & 54 & I & I & $10.3^{*}$ & 17.2 & - & + & - & $45^{\bullet}$ & $15.2^{+}$ \\
\hline 22 & $\mathrm{~F}$ & 30 & I & I & $15.4^{*}$ & $25.8^{\#}$ & + & ND & - & 29 & $7.92^{+}$ \\
\hline 23 & $\mathrm{~F}$ & 67 & 0 & II & 5.7 & $24^{\#}$ & + & + & + & 59 & $4.61^{+}$ \\
\hline 24 & $\mathrm{~F}$ & 72 & 0 & II & 0.3 & 6.7 & + & + & + & $26^{\pi}$ & $7.54^{+}$ \\
\hline 25 & $\mathrm{~F}$ & 62 & 0 & I & 6.8 & 10.4 & + & - & - & $35^{\star}$ & 1.3 \\
\hline 26 & $\mathrm{~F}$ & 51 & 0 & I & 8.9 & 17.2 & - & + & - & $15.5^{\top}$ & $8.9^{+}$ \\
\hline 27 & $\mathrm{~F}$ & 61 & 0 & 0 & 6 & 9 & + & - & - & $78^{\star}$ & $8.4^{+}$ \\
\hline 28 & $\mathrm{~F}$ & 27 & 0 & 0 & 9.2 & 9.7 & - & - & - & $58^{\bullet}$ & $6.7^{+}$ \\
\hline 29 & M & 22 & 0 & 0 & 6.4 & 12.7 & + & - & - & $55^{\circ}$ & $4.4^{+}$ \\
\hline 30 & $\mathrm{~F}$ & 27 & 0 & 0 & 6.6 & 13.6 & + & - & - & $49^{\bullet}$ & $46.9^{+}$ \\
\hline 31 & $\mathrm{~F}$ & 25 & 0 & 0 & 8.2 & 17.3 & + & - & - & $37^{\star}$ & 2.6 \\
\hline 32 & M & 59 & 0 & 0 & 6.8 & 10.6 & - & - & - & $23^{\bullet}$ & $6.5^{+}$ \\
\hline 33 & M & 22 & 0 & 0 & 7.4 & 12.3 & + & - & - & $22^{\bullet}$ & 0.9 \\
\hline 34 & $\mathrm{~F}$ & 71 & 0 & 0 & 7.1 & 9.3 & + & ND & - & 18 & $4.28^{+}$ \\
\hline 35 & M & 18 & 0 & 0 & 5.9 & 12.1 & + & - & - & 18 & 2.2 \\
\hline 36 & $\mathrm{~F}$ & 29 & 0 & 0 & ND & 11.9 & ND & ND & - & $17^{\bullet}$ & 2.97 \\
\hline 37 & $\mathrm{~F}$ & 24 & 0 & 0 & 6.7 & 7.2 & + & - & - & 8 & 2.9 \\
\hline 38 & $\mathrm{~F}$ & 24 & 0 & 0 & 3.7 & 11 & + & - & - & 7 & 1.54 \\
\hline 39 & $\mathrm{~F}$ & 75 & 0 & 0 & 7.6 & 18.6 & + & - & - & 3 & $6.39^{+}$ \\
\hline
\end{tabular}

HRCT: high-resolution computed tomography; LZM: lysozyme; SACE: serum angiotensin-converting enzyme; PPD: purified protein derivative; Ga: gallium; TBLB: transbronchial lung biopsy; BAL: bronchoalveolar lavage; M: male; F: female; ND: not done. *: serum LZM $>10.2 \mu \mathrm{g} \cdot \mathrm{mL}^{-1}$; ${ }^{\#}$ : SACE $>21.4 \mathrm{IU} \cdot \mathrm{L}^{-1} ;{ }^{*}$ : lymphocytosis $>15 \%{ }^{+}$: CD4+/CD8+ $>3.5$. Granuloma was identified by TBLB in 19 of 20 patients with type HRCT-II as opposed to one of 19 patients with type HRCT-0 and -I. Lymphocytosis $>15 \%$ in BAL was identified in all patients with type HRCT-II and in 16 of 19 patients with types HRCT-0 and -I. BAL cell lymphocytosis $(>15 \%)$ was seen in 10 of 13 patients with type HRCT-0. Serum LZM, SACE and Ga scintigram were negative in all patients with type HRCT-0.

\section{Discussion}

Ocular sarcoidosis should be defined as an ocular involvement in a patient with an established diagnosis of sarcoidosis. Ocular sarcoidosis suspects who do not fulfil the diagnostic criteria are considered to have suspected sarcoidosis. Some believe that such cases can be defined as idiopathic. Among uveitis patients, many are classified as idiopathic. Some supporting data for a diagnosis of sarcoidosis were present in the ocular sarcoidosis suspects, but the data did not fulfil the criteria for a diagnosis.

Thirty-nine consecutive patients with clinical features consistent with stages 0 , I, II and III sarcoidosis, who were highly suspected of having sarcoidosis because of ocular involvement, were studied. Precise evaluation of HRCT yielded more sensitive and appropriate information regarding pulmonary involvement associated with sarcoidosis than did the use of conventional chest radiograph film alone (fig. 1). Most patients who evidenced typical features of pulmonary involvement associated with sarcoidosis on HRCT (type HRCT-II) could be accurately shown to have sarcoid granulomas (fig. 2). HRCT assessment of lung field involvement is highly reflective of pathological involvement. It was of interest that lymphadenopathy was identified by HRCT in every patient in whom granuloma was found even if 

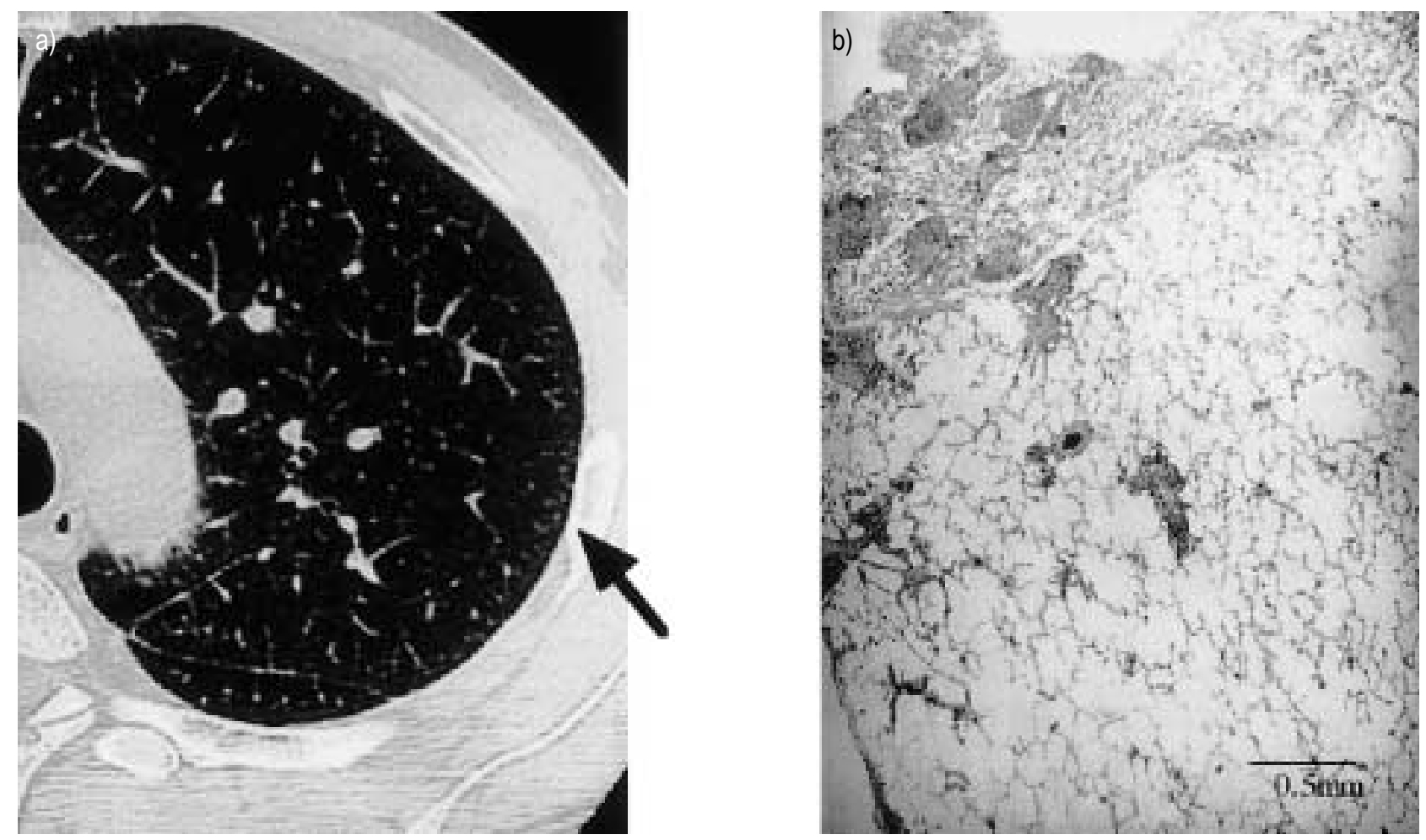

Fig. 4. - Pathological feature of subpleural granuloma oriented by high-resolution computed tomography (HRCT). a) Subtle findings of the chest HRCT, such as pleural network and subpleural small nodular shadow. Arrow indicates biopsy site under video-assisted thoracic surgery. b) Biopsy finding in the same portion as the HRCT finding shows multiple granulomas in the subpleural area (scale bar 0.5 mm).

conventional radiograph showed no abnormal shadow.

The presented HRCT findings suggested that most sarcoidosis patients were divided into two types, HRCT-0 and HRCT-II (fig. 1). It has been reported that all patients with hilar lymphadenopathy exhibited granuloma in the pulmonary region [3]; several findings might have been seen in the lung field if HRCT was used. Conversely, the results suggest that pulmonary involvement (i.e. lymphoalveolitis without granuloma formation, the HRCT-0 type) may exist in ocular sarcoidosis.

With HRCT, precise evaluation of pulmonary involvement is sometimes very difficult. Care must be taken not to overlook manifestations of ground glass opacities, pleural networks and subpleural small nodular shadows [8-10]. One of the study patients was borderline for HRCT detection of pulmonary involvement (fig. 4a), but had findings regarding pleural network and subpleural small nodular shadows that matched the pathological findings of aggregated granulomas and lymphocyte alveolitis (fig. 4b). Another sarcoidosis patient had proven extra-thoracic granuloma and was diagnosed by HRCT as having pulmonary involvement, although TBLB failed to show any evidence of granulomas in lung tissue. Radiographic assessment of pulmonary involvement with sarcoidosis may not completely reflect histological involvement, and TBLB does not correspond to radiologically occupied areas. All patients with stage II and III chest radiographs had HRCT-II scans (fig. 1). All such patients with stage II and III radiographs fall within a group of patients who have a $95 \%$ (19 of 20) chance of having a positive TBLB (fig. 2). Therefore, in this group of patients the cost of the HRCT scan could be avoided as the identical clinical information can be gleaned from chest radiographs.

BAL findings in type HRCT-0 patients were investigated further. Results showed that most patients exhibited lymphocytosis in BAL without radiographic evidence in the thoracic cage, and showed that lymphocytosis was the most sensitive indicator in patients with ocular sarcoidosis with negative results of other clinicopathological examinations (table 2) [11-16]. The cut-off value of BAL lymphocytes was selected at $15 \%$ in response to previous reports regarding normal ranges [17, 18] and based on a relevant publication [19]. All patients who were proved to have epithelioid cell granuloma had an elevated number of BAL lymphocytes $>15 \%$ (fig. 2 and table 2). Lymphocytosis $>15 \%$ in BAL, even with no evidence of granuloma, was present in association with ocular involvement in all but three of the 39 patients studied. The cut-off value for lymphocytosis in BAL should be considered for increased accuracy in the diagnosis.

Elevated SACE levels are due to secretion from the epithelioid cells comprising the granuloma [20]. The present study found no elevation of SACE levels without proof of granuloma. Since serum LZM is also secreted from activated monocytes or macrophages [21], no elevation of serum LZM was found, except in one case. In Japan, there is a requirement to receive 
bacille Calmette-Guérin (BCG) inoculations for the prevention of tuberculosis in childhood in cases of a negative PPD skin test. Therefore, almost all adults had a positive PPD skin test. Conversion of the PPD skin test will be meaningful for the cutaneous energy effect in the granulomatous pathogenesis of sarcoidosis [22]. Only two patients had a negative PPD skin test among type HRCT-0 subjects (table 2). Ga scintigram results were also negative. Since $\mathrm{Ga}$ is taken into macrophages or epithelioid cells, a Ga scintigram is not positive without granuloma formation. There are some reports that the Ga scintigram is useful for detecting granuloma $[23,24]$ in comparison with other examinations, but in this study HRCT was more sensitive and specific for the early detection of sarcoidosis (fig. 2). Ga scintigram is neither sensitive nor specific, and is very expensive; therefore, it is recommended that the use of Ga scintigram be limited to clarifying the need for therapy.

In type HRCT-0, morphological characteristics of lymphocytes in BAL were very similar to those in the histologically proven sarcoidosis patients. Further, the presented data showed a high incidence of an increased $\mathrm{CD} 4+/ \mathrm{CD} 8+$ lymphocyte ratio in the HRCT-0 type. This finding was consistent with the sarcoid lymphocytosis in BAL [25]. These ratios did not differ statistically among the types (fig. 3).

HRCT, TBLB and BAL findings in this study suggest that there is a type of ocular sarcoidosis which manifests lymphocytosis without granuloma formation. This hypothesis is also consistent with the concept of lymphocytosis prior to granuloma formation in sarcoidosis [26, 27]. Other granulomatous diseases (Crohn's disease, etc.) or infectious diseases (histoplasmosis, etc.) have been reported to be complicated by uveitis and/or lymphocytosis in BAL [28-30]. The patients in this study did not show clinical manifestations of Crohn's disease, histoplasmosis, nor other diseases. Although patients with ocular involvement are sometimes difficult to distinguish from those with other forms of idiopathic uveitis, the findings of "trabecular nodules" and "tentlike peripheral anterior synechiae" are more specific in patients with sarcoidosis [6]. These findings have encouraged the authors to diagnose sarcoidosis through looking for pulmonary involvement. In the process of following up the study patients, one patient was found to have sarcoid granuloma, but no other diseases were found. It was felt that this was important evidence to support a diagnosis of sarcoidosis.

Clearly when two separate organs contain granulomas, the diagnosis of sarcoidosis is almost certain. However, very often one organ is biopsied and clinical criteria are used to define the second organ involvement. An instrument has been developed in an attempt to standardize the diagnostic criteria for sarcoidosis [31]. According to this instrument, if granulomas have been identified in one organ and the presence of BAL lymphocytosis suggests that the lungs are "probably involved" with sarcoidosis, a diagnosis of sarcoidosis may be made. When there is difficulty in performing a biopsy in the first organ, such as with ocular sarcoidosis, a diagnosis can not be made at that time. In the face of such a dilemma, a diagnosis is needed to administer treatment. In such cases, the presence of BAL lymphocytosis may suggest that the lungs are "probably involved" with sarcoidosis if other granulomatous diseases can be excluded.

The authors conclude that high-resolution computed tomography results yield the same degree of diagnostic accuracy as transbronchial lung biopsy in ocular sarcoidosis suspects. Conversely, in patients without lung field involvement shown by highresolution computed tomography the diagnostic yield of transbronchial lung biopsy is very low. However, bronchoalveolar lavage findings showed a significant elevation of lymphocyte counts even in patients with only ocular findings. At present, a diagnosis of sarcoidosis cannot be made without granuloma, but patients must be treated with prednisolone to prevent loss of vision. It must be kept in mind that a diagnostic subgroup of patients with sarcoidosis who show ocular involvement and lymphocytosis in bronchoalveolar lavage exists.

\section{References}

1. Ohara K, Okubo A, Sasaki H, Kamata K. Intraocular manifestations of systemic sarcoidosis. Jpn J Ophthalmol 1992; 36: 452-457.

2. Koerner S, Sakowitz AJ, Appelman RI, Becker NH, Schoenbaum SW. Transbronchinal lung biopsy for the diagnosis of sarcoidosis. N Engl J Med 1975; 293: 268270.

3. Roethe RA, Fuller PB, Byrd RB, Hafermann DR. Transbronchoscopic lung biopsy in sarcoidosis. Optimal number and sites for diagnosis. Chest 1980; 77: 400-402.

4. Gilman MJ, Wang KP. Transbronchial lung biopsy in sarcoidosis. An approach to determine the optimal number of biopsies. Am Rev Respir Dis 1980; 122: 721-724.

5. Hamper UM, Fishman EK, Khouri NF, Johns CJ, Wang KP, Siegelman SS. Typical and atypical CT manifestations of pulmonary sarcoidosis. J Comput Assist Tomogr 1986; 10: 928-936.

6. Ohara K, Okubo A, Kaman K, Sasaki H, Kobayashi J, Kitamura S. Transbronchial lung biopsy in the diagnosis of suspected ocular sarcoidosis. Arch Ophthalmol 1993; 111: 642-644.

7. Anonymous. Statement on sarcoidosis. Joint Statement of the American Thoracic Society (ATS), the European Respiratory Society (ERS) and the Association of Sarcoidosis and Other Granulomatous Disorders (WASOG) adopted by the ATS Board of Directors and by the ERS Executive Committee, February 1999. Am J Respir Crit Care Med 1999; 160: 736-755.

8. Muller NL, Kullnig P, Miller RR. The CT findings of pulmonary sarcoidosis: Analysis of 25 patients. AJR Am J Roentgenol 1989; 152: 1179-1182.

9. Brainier MW, Greener P, Mompoint D, Lenoir S, de Cremoux H. Pulmonary sarcoidosis: Evaluation with High-Resolution CT. Radiology 1989; 172: 467-471.

10. Nishimura K, Itoh H, Kitaichi M, Nagai S, Izumi T. Pulmonary sarcoidosis: correlation of $\mathrm{CT}$ and histopathologic findings. Radiology 1993; 189: 105-109.

11. Schoenberger CI, Line BR, Keogh BA, Hunninghake 
GW, Crystal RG. Lung inflammation in sarcoidosis: comparison of serum angiotensin-converting enzyme levels with bronchoalveolar lavage and gallium-67 scanning assessment of the $\mathrm{T}$ lymphocyte alveolitis. Thorax 1982; 37: 19-25.

12. Viale G, Codecasa L, Bulgheroni P, et al. T-cell subsets in sarcoidosis: an immunocytochemical investigation of blood, bronchoalveolar lavage fluid, and prescalenic lymph nodes from eight patients. Hum Pathol 1986; 17: 476-481.

13. Rossi GA, Sacco O, Cosulich E, Risso A, Balbi B, Ravazzoni C. Helper T-lymphocytes in pulmonary sarcoidosis. Functional analysis of a lung T-cell subpopulation in patients with active disease. $A m$ Rev Respir Dis 1986; 133: 1086-1090.

14. Muller-Quernheim J, Pfeifer S, Strausz J, Ferlinz R. Correlation of clinical and immunologic parameters of the inflammatory activity of pulmonary sarcoidosis. Am Rev Respir Dis 1991; 144: 1322-1329.

15. Wallaert B, Ramon P, Fournier EC, Prin L, Tonnel $\mathrm{AB}$, Voisin C. Activated alveolar macrophage and lymphocyte alveolitis in extrathoracic sarcoidosis without radiological mediastinopulmonary involvement. Ann N Y Acad Sci 1986; 465: 201-210.

16. Bienfait MF, Hoogsteden HC, Baarsma GS, Adriaansen HJ, Verheijen-Breemhaar L. Diagnostic value of bronchoalveolar lavage in ocular sarcoidosis. Acta Ophthalmol 1987; 65: 745-748.

17. The BAL Cooperative Group Steering Committee. Bronchoalveolar lavage constituents in healthy individuals, idiopathic pulmonary fibrosis, and selected comparison groups. Am Rev Respir Dis 1990; 141: S169-S202.

18. Matsuda K, Satoh M, Kawanami O. Airway and alveolar inflammation assessments with bronchoalveolar lavage in various interstitial lung disorders. $J p n$ J Thoracic Dis 1992; 30: 827-834.

19. Kantrow SP, Meyer KC, Kidd P, Raghu G. The CD4/ CD8 ratio in BAL fluid is highly variable in sarcoidosis. Eur Respir J 1997; 10: 2716-2721.

20. Silverstein E, Pertschuk LP, Friedland J. Immunofluorescent localization of angiotensin converting enzyme in epithelioid and giant cells of sarcoidosis granuloma. Proc Natl Acad Sci USA 1979; 76: 66466664.

21. Pascual RS, Gee JBL, Finch SC. Usefulness of serum lysozyme measurement in diagnosis and evaluation of sarcoidosis. N Engl J Med 1973; 289: 1074-1076.

22. Mishra BB, Poulter LW, Janossy G, James DG. The distribution of lymphoid and macrophage like cell subsets of sarcoid and Kveim granulomata: possible mechanism of negative PPD reaction in sarcoidosis. Clin Exp Immunol 1983; 54: 705-715.

23. Duffy GJ, Thirumurthi K, Casey $\mathrm{M}$, et al. Semiquantitive gallium-67 lung scanning as a measure of the intensity of alveolitis in pulmonary sarcoidosis. Eur J Nucl Med 1986; 12: 187-191.

24. Myslivecek M, Husak V, Kolek V, Budikova M, Koranda P. Absolute quantitation of gallium 67 citrate accumulation in the lungs and its importance for the evaluation of disease activity in pulmonary sarcoidosis. Eur J Nucl Med 1992; 19: 1016-1022.

25. Costabel U, Zaiss AW, Guzman J. Sensitivity and specificity of BAL findings in sarcoidosis. Sarcoidosis 1992; 9: Suppl. 1, 211-214.

26. Keogh BA, Hunninghake GW, Line BR, Crystal RG. The alveolitis of pulmonary sarcoidosis. Evaluation of natural history and alveolitis dependent changes in lung function. Am Rev Respir Dis 1983; 28: 256265.

27. Rossman MD, Dauber JH, Daniele RP. Identification of activated T cells in sarcoidosis. Am Rev Respir Dis 1978; 117: 713-720.

28. Wallaert B, Colombel JF, Tonnel AB, et al. Evidence of lymphocyte alveolitis in Crohn's disease. Chest 1985; 87: 363-367.

29. Kayser K, Probst F, Gabius HJ, Muller KM. Are there characteristic alterations in lung tissue associated with Crohn's disease? Path Res Pract 1990; 186: 485490.

30. Nuzzi R, Amerio G. Uveitis and systemic disease. Minerva Med 1992; 83: 239-248.

31. Judson MA, Baughman RP, Teistein AS, Terrin ML, Yeager H Jr. Defining organ involvement in sarcoidosis: the ACCESS proposed instrument. ACCESS Research Group. A Case Control Etiologic Study of Sarcoidosis. Sarcoidosis Vasc Diffuse 1999; 16: 75-86. 